\title{
MicroRNAs as Potential Markers of Parenteral Nutrition-Associated Liver Disease in Adult Patients
}

\author{
M. CAHOVÁ ${ }^{1}$, H. DAŇKOVA ${ }^{1}$, M. HECZKOVÁ ${ }^{1}$, M. BRÁTOVÁ ${ }^{1}$, N. ĎÁSKOVÁ ${ }^{1}$, \\ H. BAŠTOVÁ ${ }^{2}$, J. GOJDA ${ }^{3}$, P. WOHL ${ }^{2}$ \\ ${ }^{1}$ Center for Experimental Medicine, Institute for Clinical and Experimental Medicine, Prague, \\ ${ }^{2}$ Diabetes Center, Institute for Clinical and Experimental Medicine, Prague, ${ }^{3}$ Second Department of \\ Medicine, University Hospital Královské Vinohrady and Third Faculty of Medicine, Charles \\ University, Prague, Czech Republic
}

Received December 11, 2018

Accepted March 19, 2019

Epub Ahead of Print June 6, 2019

\begin{abstract}
Summary
Parenteral nutrition-associated liver disease (PNALD) is a severe complication in patients completely dependent on parenteral nutrition (PN). The gold diagnostic standard, liver biopsy, is associated with significant health risk and therefore its use is limited. MicroRNAs (miRNAs) are small non-coding regulatory RNA molecules with highly tissue-specific expression and the secreted miRNAs may serve as non-invasive diagnostic biomarkers. The aim of this study was to evaluate the expression of a panel of specific miRNAs associated with liver diseases of different origin in $\mathrm{PN}$-dependent adult patients in order to design miRNA panel enabling to precise monitoring of PNALD progression. Twelve PN-dependent patients with short bowel syndrome (SBS) were monitored on three/four-month basis for up to 24 months. Forty-five age- and sex-matched subjects without any known liver pathology served as controls. Specific miRNAs expression was determined by RT-qPCR using TaqMan probes (Thermofisher). Liver function test parameters were determined in certified clinical laboratories. Six of the tested miRNAs exhibited significantly altered expression compared with healthy controls, three of them (MIR122, MIR1273g, and MIR500a) were upregulated while three were down-regulated (MIR505, MIR199a, MIR139). MIR122 positively correlated with serum AST and ALT activities while MIR1273g positively correlated with serum CRP concentration and GGT activity. MIR505, MIR199a, and MIR139 negatively correlated with serum GGT activity. Fluctuation of these parameters well paralleled serum miRNA concentrations in all patients throughout the whole
\end{abstract}

observation period. We identified six miRNAs whose serum concentrations are significantly altered in PN-dependent patients with PNALD and correlate with markers of inflammation, cholestasis or hepatic injury. Their reliability as markers of PNALD progression needs to be further evaluated.

\section{Key words}

miRNA • Parenteral nutrition-associated liver disease • Biomarker

\section{Corresponding author}

M. Cahova, Centre of Experimental Medicine, Institute for Clinical and Experimental Medicine, Videnska 1958, 14021 Prague 4, Czech Republic. E-mail: monika.cahova@ikem.cz

Long-term administration of total parenteral nutrition $(\mathrm{PN})$ is often associated with the development of parenteral nutrition-associated liver disease (PNALD). The origin of this pathologic condition is multifactorial with numerous contributing factors, such as sepsis, intestinal inflammation, cholangitis, cholelithiasis, bacterial translocation, short bowel syndrome, the disturbance of hepato-biliary circulation, the lack of enteral nutrition, etc. PNALD clinical manifestations which range from steatosis, cholestasis, gallbladder sludge/stones, fibrosis, and cirrhosis - can occur separately or in combination (Drongowski et al. 2009, Luman et al. 2002). The history of PNALD in adult patients is characterized by elevated liver enzymes in 
association with steatosis lasting for years, followed by steatohepatitis, cholestatic hepatitis as well as fibrosis and cirrhosis (Cahova et al. 2017). The exact staging of the disease progression is necessary for the determination of the right prognosis and efficient treatment, including the indication for the intestine transplantation. It was repeatedly shown that liver tests alone are not sensitive enough for the diagnosis (Klek et al. 2016). Therefore, a liver biopsy remains the gold diagnostic procedure. Nevertheless, liver biopsy is associated with significant health risk and therefore its use is limited. There is an urgent need for seeking novel diagnostic tools for PNALD. These would help to optimize existing PN administration regimen/composition in order to delay or even prevent the development of PNALD. The aim of our study was to determine serum concentrations of selected miRNAs associated with liver pathologies of different origin in a cohort of adult PN-dependent patients in order to design miRNA panel enabling to precise monitoring of PNALD progression.

miRNAs are small endogenous RNA molecules that post-transcriptionally regulate gene expression by preferentially targeting the 3 '-untranslated region of specific mRNA (Marin et al. 2014). The specific miRNA/mRNA interaction typically results in negative regulation of the expression of the protein encoded by target mRNA (Grimson et al. 2007). The occurrence of miRNAs is not restricted into intracellular space, in contrast, they are found in extracellular body fluids like blood, milk, urine, cerebral spinal fluid, semen, saliva and bile (Shigehara et al. 2011). Extracellular miRNAs are quite stable (Gori et al. 2014). Many of the circulating miRNAs are highly tissue-specific (Ninomiya et al. 2013) and emerging evidence shows that they can serve as non-invasive diagnostic biomarkers for various diseases, including non-alcoholic fatty liver disease (Yamada et al. 2013), steatohepatitis (Jin et al. 2012), biliary diseases (Munoz-Garrido et al. 2012) or hepatocellular cancer (Gailhouste et al. 2013).

We performed an extensive computer-based search of published articles in PubMed to identify relevant studies on the usefulness of serum miRNAs as non-invasive biomarkers for the detection of liver pathologies. The used Medical Subject Headings terms and keywords were "miRNA", "biomarker", „liver disease", "PNALD", "cholestasis" and "NASH". We found 52 miRNAs proposed as putative biomarkers of liver injury (Table 1) that were further analyzed in a cohort of adult patients with chronic intestinal failure.
The discovery cohort consisted of 12 subjects with short bowel syndrome of different etiologies who were repeatedly monitored on three/four-month basis for up to 24 months. Underlying cause of SBS were mesenteric ischemia $(n=4)$, Crohn disease $(n=1)$, ulcerative colitis $(n=1)$, Gardner syndrome $(n=1)$, post radiation enteritis $(n=3)$, postsurgical adhesion $(n=1)$ and trauma $(\mathrm{n}=1)$. Control cohort included 45 apparently healthy age- and sex-matched subjects without any known liver pathology. Blood sample with no additives was taken between 7-8 a.m. in a fasting state and it was left at room temperature for $30 \mathrm{~min}$. Then it was centrifuged twice for $3000 \mathrm{~g}, 15 \mathrm{~min}, 4{ }^{\circ} \mathrm{C}$, serum removed to the new tube and centrifuged again $3000 \mathrm{~g}$, $10 \mathrm{~min}, 4{ }^{\circ} \mathrm{C}$ in order to remove any blood elements. The serum was aliquoted and stored at $-80{ }^{\circ} \mathrm{C}$ until analysis. miRNA extraction was performed using miRCURY RNA isolation kit - biofluids (Exiqon) with RNA Carrier MS2 $10 \mathrm{ng} / \mu \mathrm{l}$ (Roche). miRNA detection system included specific Taqman MicroRNA Reverse Transcription kit and TaqMan microRNA assays (Thermofisher Scientific). The PCR reaction was performed on ViiA7 Real-Time PCR system (Thermofisher Scientific). The specific miRNA expression was normalized to Stock Serum/Plasma spike-in control Caenorhabditis elegans MIR39 (cel miR-39-3p), $2 \times 10^{6}$ molecules per sample (Qiagen). The data are expressed as $2^{\Delta \mathrm{Ct}}\left(\Delta \mathrm{Ct}=\mathrm{Ct}_{\text {miRNA }}-\right.$ $\left.\mathrm{Ct}_{\text {cel miR-39) }}\right)$ and presented as a median and interquartile range. Statistical analysis was performed using the Kruskal-Wallis test. Differences were considered statistically significant at the level of $p<0.05$. Spearman's rank correlation coefficient was used to assess the correlation between the studied variables.

SBS patients represent a highly diverse cohort with respect to the primary diagnosis, duration of PN-dependence or age. Most of the patients (11 out of 12) exhibited chronically abnormal liver function tests (Table 2). Among all miRNAs tested, six exhibited significantly altered expression compared with healthy controls. Three of them (MIR122, MIR1273g, and MIR500a) were upregulated while three were downregulated (MIR505, MIR199a, MIR139) in SBS patients. MIR122 positively correlated with s-AST and s-ALT activities while MIR1273g positively correlated with s-CRP concentration and with s-GGT activity. MIR505, MIR199a, and MIR139 negatively correlated with s-GGT activity (Table 3). Fluctuation of these parameters well paralleled serum miRNA concentrations in all patients throughout the whole observation period. 
Table 1. miRNAs identified as potential biomarkers of liver injury.

\begin{tabular}{|c|c|c|c|c|}
\hline $\begin{array}{l}\text { HGNC } \\
\text { ID }\end{array}$ & & $\begin{array}{l}\text { TaqMan } \\
\text { assay ID }\end{array}$ & & reference \\
\hline 31476 & MIRLET7A & 000377 & liver fibrosis & 10.1371/journal.pone.004836 \\
\hline \multirow[t]{2}{*}{31479} & MIRLET7b-5p & 002619 & APAP-induced liver injury & $10.1093 /$ toxsci/kfy 200 \\
\hline & MIR16 & 000391 & NAFLD, NASH & 10.1371/journal.pone.0023937 \\
\hline 31575 & MIR19b & 002425 & liver fibrosis & 10.1371/journal.pone.0048366 \\
\hline \multirow[t]{2}{*}{31586} & MIR21 & 000397 & APAP-induced liver injury & 10.1093/toxsci/kfy200 \\
\hline & & & liver inflammation & 10.1371/journal.pone.0023937 \\
\hline \multirow[t]{2}{*}{31599} & MIR22 & 002301 & liver inflammation & 10.1371/journal.pone.0048366 \\
\hline & MIR24 & 000402 & liver fibrosis & 10.1371/journal.pone. 0048366 \\
\hline 31616 & MIR29A & 002112 & lower circulating levels in & 10.1002/hep.23922 \\
\hline 31619 & MIR29B1 & 000413 & patients with liver fibrosis & \\
\hline 31621 & MIR29C & 000587 & & \\
\hline \multirow[t]{2}{*}{31625} & MIR30B & 000602 & primary biliary cirrhosis & 10.1371/journal.pone.0066086 \\
\hline & & & NAFLD & 10.1016/j.hep.2018.08.008 \\
\hline 31634 & MIR33a & 002135 & primary biliary cirrhosis & 10.1371/journal.pone.0066086 \\
\hline 32791 & MIR33b & 002085 & NAFLD & 10.1016/j.hep.2018.08.008 \\
\hline \multirow[t]{3}{*}{31635} & MIR34a & 000426 & APAP-induced liver injury & $10.1002 /$ jat.3722 \\
\hline & & & liver inflammation & 10.1371/journal.pone.0048366 \\
\hline & & & NAFLD & 10.1016/j.hep.2018.08.008 \\
\hline 31648 & MIR96 & 000186 & apoptosis, necrosis & $10.1080 / 1354750 X .2018 .1528631$ \\
\hline 31650 & MIR99A & 000435 & NASH & 10.4254/wjh.v6.i8.613 \\
\hline 31495 & MIR106b & 000442 & liver fibrosis & 10.1371/journal.pone.0048366 \\
\hline \multirow[t]{5}{*}{31501} & MIR122 & 002245 & drug-induced liver injury & 10.1093/toxsci/kfy200 \\
\hline & & & apoptosis, necrosis & 10.1080/1354750X.2018.1528631 \\
\hline & & & oxidative stress & $10.3164 /$ jcbn. $17-123$ \\
\hline & & & NASH & 10.1016/j.cca.2013.05.021 \\
\hline & & & NAFLD & 10.1371/journal.pone.0153497 \\
\hline 31505 & MIR125 & 002198 & NAFLD & 10.1136/gutjnl-2014-306996 \\
\hline \multirow[t]{2}{*}{31514} & MIR130a & 000454 & apoptosis, necrosis & $10.1080 / 1354750 X .2018 .1528631$ \\
\hline & & & liver inflammation & 10.1371/journal.pone.0048366 \\
\hline \multirow[t]{2}{*}{31526} & MIR139 & 001096 & primary biliary cirrhosis & 10.1371/journal.pone.0066086 \\
\hline & & & NAFLD, NASH & 10.1038/ijo.2017.21 \\
\hline 31530 & MIR143-3p & 002249 & cholestasis & $10.1093 /$ toxsci/kfy 200 \\
\hline \multirow[t]{2}{*}{32079} & MIR146B & 001097 & NAFLD, NASH & 10.1136/gutjnl-2015-309456 \\
\hline & & & & 10.4254/wjh.v6.i8.613 \\
\hline \multirow[t]{2}{*}{31537} & MIR150 & 002637 & NAFLD, NASH & 10.1136/gutjnl-2015-309456 \\
\hline & & & & 10.1016/j.bbrc.2017.10.149 \\
\hline 31762 & MIR151a & 002642 & APAP-induced liver injury & 10.1002/jat.3722 \\
\hline
\end{tabular}


Table 1., continued.

\begin{tabular}{|c|c|c|c|c|}
\hline \multirow[t]{2}{*}{31549} & \multirow[t]{2}{*}{ MIR181a } & \multirow[t]{2}{*}{000480} & NAFLD progression & 10.1016/j.taap.2012.04.018 \\
\hline & & & liver cirrhosis & 10.1016/j.bbrc.2012.03.025 \\
\hline 31554 & MIR183 & 002269 & apoptosis, necrosis & 10.1080/1354750X.2018.1528631 \\
\hline 31560 & MIR190 & 000489 & cholestasis & 10.1097/MOG.0000000000000051 \\
\hline \multirow[t]{3}{*}{31562} & \multirow[t]{3}{*}{ MIR192-5p } & \multirow[t]{3}{*}{000491} & drug-induced liver injury & 10.1093/toxsci/kfy200 \\
\hline & & & oxidative stress & $10.3164 /$ jcbn. $17-123$ \\
\hline & & & NAFLD, NASH & 10.1016/j.hep.2018.08.008 \\
\hline \multirow[t]{3}{*}{31563} & \multirow[t]{2}{*}{ MIR193a } & \multirow[t]{2}{*}{002281} & APAP-induced liver injury & $10.1002 /$ jat.3722 \\
\hline & & & liver inflammation & 10.1371/journal.pone. 0048366 \\
\hline & MIR194 & 000493 & APAP-induced liver injury & 10.1002/jat.3722 \\
\hline 31567 & MIR196 & 241070_mat & apoptosis, necrosis & 10.1080/1354750X.2018.1528631 \\
\hline \multirow[t]{2}{*}{31569} & \multirow[t]{2}{*}{ MIR197 } & \multirow[t]{2}{*}{ 474626_mat } & primary biliary cirrhosis & 10.1371/journal.pone. 0066086 \\
\hline & & & liver inflammation & 10.1371/journal.pone.0048366 \\
\hline \multirow[t]{2}{*}{31571} & \multirow[t]{2}{*}{ MIR199a } & \multirow[t]{2}{*}{000498} & alcoholic liver disease & 10.3390/ijms 17030280 \\
\hline & & & liver fibrosis & $10.1038 /$ nrgastro. 2013.87 \\
\hline \multirow[t]{3}{*}{31579} & \multirow[t]{2}{*}{ MIR200B } & \multirow[t]{2}{*}{002274} & liver inflammation & $10.1016 / \mathrm{S} 0168-8278(15) 31170-3$ \\
\hline & & & steatosis & 10.18632/oncotarget.9183 \\
\hline & MIR218a-5p & 000521 & cholestasis & 10.1093/toxsci/kfy200 \\
\hline \multirow[t]{2}{*}{31601} & \multirow[t]{2}{*}{ MIR221 } & \multirow[t]{2}{*}{002096} & liver fibrosis & $10.1038 /$ nrgastro. 2013.87 \\
\hline & & & hepatocellular carcinoma & 10.1073/pnas.0907904107 \\
\hline 31771 & MIR320-3p & 002230 & steatosis & 10.1093/toxsci/kfy200 \\
\hline 31868 & MIR375 & 000564 & NASH & 10.1136/gutjnl-2014-306996 \\
\hline 32053 & MIR451 & 001141 & NAFLD & 10.1016/j.cca.2013.05.021 \\
\hline 32134 & MIR500a & 002428 & primary biliary cirrhosis & 10.1371/journal.pone.0066086 \\
\hline 32140 & MIR505 & 002087 & primary biliary cirrhosis & 10.1371/journal.pone.0066086 \\
\hline 32827 & \multicolumn{2}{|l|}{ MIR571 } & $\begin{array}{l}\text { correlates with disease stages } \\
\text { during alcoholic or HCV- } \\
\text { induced liver cirrhosis }\end{array}$ & 10.1371/journal.pone.0032999 \\
\hline 32828 & MIR572 & 001614 & NASH & 10.3748/wjg.v18.i37.5188 \\
\hline 32831 & MIR575 & 001617 & NASH & 10.3748/wjg.v18.i37.5188 \\
\hline 32894 & MIR638 & 001582 & NASH & 10.3748/wjg.v18.i37.5188 \\
\hline 32915 & MIR659 & 001514 & liver inflammation & 10.1371/journal.pone.0048366 \\
\hline 37316 & MIR711 & 241090_mat & liver inflammation & 10.1371/journal.pone.0048366 \\
\hline 33658 & MIR744 & 002324 & NASH & 10.3748/wjg.v18.i37.5188 \\
\hline \multirow[t]{3}{*}{33923} & MIR-1224-5p & 002752 & oxidative stress & $10.3164 / j \mathrm{cbn} .17-123$ \\
\hline & MIR1273g & 462577_mat & primary biliary cirrhosis & 10.1371/journal.pone.0066086 \\
\hline & MIR1274B & 002884 & liver inflammation & 10.1371/journal.pone. 0048366 \\
\hline
\end{tabular}


MIR122 is highly enriched in the liver but absent in other tissues (Lagos-Quintana et al. 2002). MIR122 participates on the regulation of the expression of enzymes involved in crucial metabolic pathways in the liver including glycolysis and gluconeogenesis, carbohydrate digestion and absorption, glucagon signaling pathway, starch and sucrose metabolism, cholesterol synthesis or iron homeostasis (Joppling 2012). Several lines of evidence indicate that it functions as a tumor suppressor (Bai et al. 2009). Roderburg et al. (2015) showed that serum MIR122 concentrations were strongly elevated in mice after hepatic ischemia/reperfusion injury, as well as in the cellular supernatants in an in vitro model of hepatocyte injury, supporting the hypothesis that the passive release of MIR122 represents a surrogate for hepatocyte death in liver injury. This finding corresponds with our observation that serum MIR122 levels correlate with ALT and AST concentrations. Taken together, MIR122 levels may serve as an independent marker of ongoing liver injury and hepatic cell death.

Serum content of all other miRNAs deregulated in PN-dependent patients significantly correlated with GGT serum activity, which is a marker of cholestasis. To our knowledge, this association has not been described yet. Three of these miRNAs (MIR199a, MIR505, and MIR139) were described as tumor suppressors and their down-regulation is associated with disease progression.

Table 2. Clinical characteristics of patients.

\begin{tabular}{|c|c|c|c|c|c|c|c|c|c|c|c|c|c|c|}
\hline 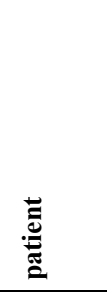 & 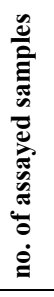 & 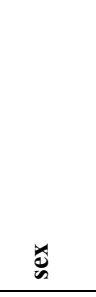 & 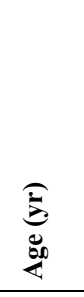 & $\begin{array}{l}\sum_{2}^{2} \\
0 \\
0 \\
0 \\
0\end{array}$ & 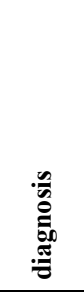 & 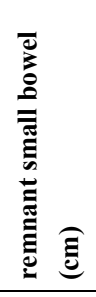 & 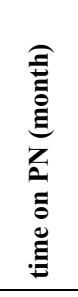 & 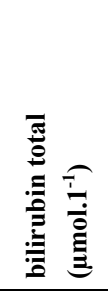 & 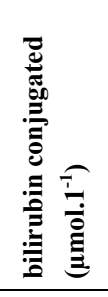 & 章 & 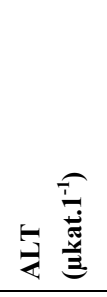 & 焉 & 包 & 晑 \\
\hline 1 & 6 & M & 36 & I & 1 & 50 & 8 & $\begin{array}{l}60 * \\
(37)\end{array}$ & $\begin{array}{c}32.8^{*} \\
(22)\end{array}$ & $\begin{array}{l}1.4^{*} \\
(0.7)\end{array}$ & $\begin{array}{l}2.6^{*} \\
(1.3)\end{array}$ & $\begin{array}{l}4.1 * \\
(1.4)\end{array}$ & $\begin{array}{l}2.4^{*} \\
(1.1)\end{array}$ & $\begin{array}{c}7.7 * \\
(54.9)\end{array}$ \\
\hline 2 & 4 & F & 71 & I & 2 & 100 & 87 & $\begin{array}{l}28^{*} \\
(15)\end{array}$ & $\begin{array}{l}12^{*} \\
(3)\end{array}$ & $\begin{array}{c}0.5 \\
(0.08)\end{array}$ & $\begin{array}{c}0.5 \\
(0.16)\end{array}$ & $\begin{array}{l}2.2^{*} \\
(0.2)\end{array}$ & $\begin{array}{l}1.9^{*} \\
(0.2)\end{array}$ & $\begin{array}{c}0.6 \\
(0.2)\end{array}$ \\
\hline 3 & 1 & F & 52 & I & 3 & 200 & 84 & 9.4 & n.d. & 0.4 & 0.5 & $1.8^{*}$ & $0.6^{*}$ & 5.3 \\
\hline 4 & 3 & F & 64 & I & 5 & 40 & 96 & $\begin{array}{c}19.5^{*} \\
(6.3)\end{array}$ & $\begin{array}{l}11.0 * \\
(2.4)\end{array}$ & $\begin{array}{l}0.7^{*} \\
(0.2)\end{array}$ & $\begin{array}{l}0.6^{*} \\
(0.2)\end{array}$ & $\begin{array}{l}3.6^{*} \\
(2,3)\end{array}$ & $\begin{array}{c}1.3 * \\
0.3)\end{array}$ & $\begin{array}{l}75.8^{*} \\
(39.8)\end{array}$ \\
\hline 5 & 1 & M & 64 & I & 7 & $?$ & 13 & 19.9 & 11.0 & $0.6^{*}$ & $0.7^{*}$ & $5.1^{*}$ & $2.7^{*}$ & 2.3 \\
\hline 6 & 4 & $\mathrm{~F}$ & 53 & I & 5 & 120 & 16 & $\begin{array}{c}7 \\
(2.6)\end{array}$ & $\begin{array}{c}3.7 \\
(1.5)\end{array}$ & $\begin{array}{l}0.7^{*} \\
(0.3)\end{array}$ & $\begin{array}{l}0.5^{*} \\
(0.3)\end{array}$ & $\begin{array}{l}6.5^{*} \\
(1.7)\end{array}$ & $\begin{array}{l}1.6^{*} \\
(0.6)\end{array}$ & $\begin{array}{l}31 * \\
(41)\end{array}$ \\
\hline 7 & 5 & F & 38 & II & 4 & 15 & 27 & $\begin{array}{c}8.7 \\
(3.4)\end{array}$ & $\begin{array}{c}3.4 \\
(0.9)\end{array}$ & $\begin{array}{c}0.5 \\
(0.3)\end{array}$ & $\begin{array}{c}0.7^{*} \\
(0.62)\end{array}$ & $\begin{array}{l}1.8^{*} \\
(0.7)\end{array}$ & $\begin{array}{c}0.3 \\
(0.9)\end{array}$ & $\begin{array}{c}3.7 \\
(4.3)\end{array}$ \\
\hline 8 & 4 & F & 68 & II & 1 & 40 & 88 & $\begin{array}{l}14.6 \\
(3.9)\end{array}$ & $\begin{array}{c}7.6 \\
(1.7)\end{array}$ & $\begin{array}{l}0.5^{*} \\
(0.2)\end{array}$ & $\begin{array}{c}0.7^{*} \\
(0.41)\end{array}$ & $\begin{array}{l}2.2 * \\
(3.3)\end{array}$ & $\begin{array}{l}2.4^{*} \\
(1.7)\end{array}$ & $\begin{array}{l}11.4 * \\
(19.4)\end{array}$ \\
\hline 9 & 5 & F & 40 & II & 1 & 30 & 61 & $\begin{array}{c}27 * \\
(18.8)\end{array}$ & $\begin{array}{c}10.3 * \\
(4.5)\end{array}$ & $\begin{array}{c}0.4 \\
(0.2)\end{array}$ & $\begin{array}{c}0.6^{*} \\
(0.19)\end{array}$ & $\begin{array}{l}3.0^{*} \\
(1.4)\end{array}$ & $\begin{array}{c}0.3 \\
(0.2)\end{array}$ & $\begin{array}{c}0.2 \\
(0.1)\end{array}$ \\
\hline 10 & 3 & F & 37 & II & 1 & 30 & 52 & $\begin{array}{c}6.1 \\
(1.9)\end{array}$ & $\begin{array}{c}2.9 \\
(0.4)\end{array}$ & $\begin{array}{c}0.3 \\
(0.02)\end{array}$ & $\begin{array}{c}0.53 \\
(0.08)\end{array}$ & $\begin{array}{c}1.6 \\
(0.1)\end{array}$ & $\begin{array}{c}0.5 \\
(0.1)\end{array}$ & $\begin{array}{c}0.5 \\
(0.4)\end{array}$ \\
\hline 11 & 1 & M & 21 & II & 6 & 80 & 18 & 6.7 & 2.7 & $0.7^{*}$ & $0.9^{*}$ & 0.7 & 0.3 & 2.5 \\
\hline 12 & 1 & $\mathrm{~F}$ & 50 & II & 5 & $?$ & 84 & 6.2 & 3.1 & $1.8^{*}$ & $1.1^{*}$ & $2.3 *$ & 0.4 & $13.2 *$ \\
\hline controls & & $\begin{array}{c}29 \mathrm{~F} / 1 \\
6 \mathrm{M}\end{array}$ & $\begin{array}{c}40 \\
(25)\end{array}$ & N/A & N/A & N/A & N/A & $\begin{array}{c}7.8 \\
(1.9)\end{array}$ & $\begin{array}{c}2.8 \\
(0.7)\end{array}$ & $\begin{array}{c}0.3 \\
(0.4)\end{array}$ & $\begin{array}{c}0.4 \\
(0.6)\end{array}$ & $\begin{array}{c}0.9 \\
(0.5)\end{array}$ & $\begin{array}{c}0.2 \\
(0.2)\end{array}$ & $\begin{array}{c}1.8 \\
(2.1)\end{array}$ \\
\hline
\end{tabular}

SBS type I: end-ostomy, SBS type II: bowel in continuity. Diagnoses: 1 mesenteric ischemia, 2 Crohn disease, 3 ulcerative colitis, 4 Gardner syndrome, 5 post-radiation enteritis, 6 post-surgical adhesion, 7 trauma. AST aspartate transaminase; ALT alanine transaminase; ALP alkaline phosphatase; GGT gamma-glutamyl transpeptidase; CRP C-reactive protein. When applicable, data are given as a median and interquartile range. Values marked with $*$ were above the normal range in more than half samples during the observation period. 
Table 3. Content of selected miRNA in the serum of PN-dependent patients and healthy controls.

\begin{tabular}{lcccccccc}
\hline \multirow{2}{*}{ symbol } & \multirow{2}{*}{ HGNC ID } & \multirow{2}{*}{ fold change } & p-value & \multicolumn{4}{c}{ Spearman's correlation coefficient } \\
& & & & AST & ALT & ALP & GGT & CRP \\
\hline \multirow{2}{*}{ MIR122 } & \multirow{2}{*}{1501} & $1.7(1.8)$ & 0.024 & $0.685^{*}$ & $0.873^{*}$ & 0.305 & 0.254 & 0.008 \\
MIR1273G & - & $3.7(6.9)$ & 0.003 & -0.048 & -0.131 & 0.316 & $0.761^{*}$ & $0.531^{*}$ \\
MIR500A & 32134 & $2.1(1.9)$ & 0.049 & -0.157 & -0.126 & -0.038 & $-0.463^{*}$ & -0.021 \\
MIR199A1 & 31571 & $0.3(0.5)$ & $2.7 \times 10^{-5}$ & -0.070 & -0.085 & -0.246 & $-0.510^{*}$ & -0.325 \\
MIR505 & 32140 & $0.1(0.1)$ & $2.4 \times 10^{-12}$ & 0.336 & 0.250 & -0.323 & $0.697^{*}$ & 0.056 \\
MIR139 & 31526 & $0.5(0.3)$ & 0.025 & -0.195 & -0.254 & -0.044 & $-0.599^{*}$ & -0.338 \\
\hline
\end{tabular}

Fold change is calculated as the ratio of normalized Ct values (patients vs a median of control cohort) and expressed as a median and interquartile range (IQR). $p$-value shows the significance of the difference between control and patient cohorts (Kruskal-Wallis test with Bonferroni correction). Spearman's correlation coefficient: values marked by * are statistically significant at the level $p<0.05$.

MIR505 is down-regulated in the serum of pancreatic cancer patients (Schultz et al. 2014) and patients with hepatocellular carcinoma (HCC) (Li et al. 2015) as well as in serum of patients with primary biliary cirrhosis (Ninomiya et al. 2013). In hepatoma cell lines downregulation of MIR505 promoted proliferation, invasion and epithelial-mesenchymal transition (Lu et al. 2016). Serum means values of MIR199a were significantly decreased among HCC patients (Kamel et al. 2016, Yin et al. 2015) and served as a predictor of hepatitis B- or hepatitis C-related HCC (Fiorino et al. 2016). MIR139 suppresses tumor growth and metastasis in HCC and its decreased serum levels may serve as biomarker of this pathology (Zou et al. 2018). MIR500a promotes the progression of hepatocellular carcinoma and enhances HCC (Bao et al. 2018, Jiang et al. 2017, Zhao et al. 2017). The biological function of MIR1273g has not been described yet but the increased MIR1273g content was observed in mice pancreatic cancer tissue (Rachagani et al. 2015) and in human colorectal carcinoma tissue (Vishnubalaji et al. 2015). Interestingly, the expression pattern of all these five miRNAs in patients' cohorts (down MIR199a, MIR505, MIR139; up MIR500a,
MIR1273g) follows the signature characteristic of hepatocellular or pancreatic cancer. In our cohort of patients, there was only one case with diagnosed GIT-related cancer (Gardner syndrome) but our data suggest the increased risk of increased proliferation and possible malignant transformation in the liver of PN-dependent patients.

In conclusion, we identified a panel of six miRNAs differently expressed in sera of PN-dependent patients with abnormal liver function tests compared with healthy controls. These miRNAs correlated with liver injury and hepatic cell death (MIR122), cholestasis (MIR505, MIR199a, MIR139, MIR500a, MIR1273g) or inflammation (MIR1273g). This study suggests that specific miRNAs profile in serum has potential as a diagnostic biomarker of PNALD progression.

\section{Conflict of Interest}

There is no conflict of interest.

\section{Acknowledgements}

Supported by Ministry of Health of the Czech Republic, grant no. 15-28745A AZV MZ CR.

\section{References}

BAI S, NASSER MW, WANG B, HSU SH, DATTA J, KUTAY H: MicroRNA-122 inhibits tumorigenic properties of hepatocellular carcinoma cells and sensitizes these cells to sorafenib. J Biol Chem 284: 32015-32027, 2009.

BAO L, ZHANG M, HAN S, ZHAN Y, GUO W, TENG F: MicroRNA-500a Promotes the Progression of Hepatocellular Carcinoma by Post-Transcriptionally Targeting BID. Cell Physiol Biochem 47: 2046-2055, 2018.

CAHOVA M, BRATOVA M, WOHL P: Parenteral Nutrition-Associated Liver Disease: The Role of the Gut Microbiota. Nutrients 9: 2017. doi: 10.3390/nu9090987. 
DRONGOWSKI RA, CORAN AG: An analysis of factors contributing to the development of total parenteral nutritioninduced cholestasis. JPEN J Parenter Enteral Nutr 13: 586-589, 1989.

FIORINO S, BACCHI-REGGIANI ML, VISANI M, ACQUAVIVA G, FORMELLI A, MAETTI M: MicroRNAs as possible biomarkers for diagnosis and prognosis of hepatitis B- and C-related-hepatocellular-carcinoma. World J Gastroenterol 22: 3907-3936, 2016.

GAILHOUSTE L, GOMEZ-SANTOZ L, OCHIYA T: Potential applications of miRNAs as diagnostic and prognostic markers in liver cancer. Front Biosci (Landmark Ed) 18: 199-223, 2013.

GORI M, ARCIELLO M, BALSANO C: MicroRNAs in nonalcoholic fatty liver disease: novel biomarkers and prognostic tools during the transition from steatosis to hepatocarcinoma. Biomed Res Int 2014: 741465.

GRIMSON A, FARH KK, JOHNSTON WK, GARRETT-ENGELE P, LIM LP, BARTEL DP: MicroRNA targeting specificity in mammals: determinants beyond seed pairing. Mol Cell 27: 91-105, 2007.

JIANG C, LONG J, LIU B, XU M, WANG W, XIE X: JmiR-500a-3p promotes cancer stem cells properties via STAT3 pathway in human hepatocellular carcinoma. J Exp Clin Cancer Res 36: 99, 2017.

JIN X, CHEN YP, KONG M, ZHENG L, YANG YD, LI YM: Transition from hepatic steatosis to steatohepatitis: unique microRNA patterns and potential downstream functions and pathways. J Gastroenterol Hepatol 27: 331-340 2012.

JOPLLING C: Liver-specific microRNA-122: Biogenesis and function. RNA Biol 9: 137-142, 2012.

KAMEL RR, AMR KS, AFIY M, ELHOSARY YA, HEGAZY AE, FAHIM HH: Relation between microRNAs and apoptosis in hepatocellular carcinoma. Open Access Maced J Med Sci 4: 31-37, 2016.

KLEK S, FORBES A, GABE S, HOLST M, WANTEN G, IRTEN O: Management of acute intestinal failure: A position paper from the European Society for Clinical Nutrition and Metabolism (ESPEN) Special Interest Group. Clin Nutr 35: 1209-1218, 2016.

LAGOS-QUINTANA M, RAUHUT R, YALCIN A, MEYER J, LENDECKEL W, TUSCHL T: Identification of tissue-specific microRNAs from mouse. Curr Biol 12: 735-739, 2002.

LI J, QIN S, XU R, YAU TC, MA B, PAN H: Regorafenib plus best supportive care versus placebo plus best supportive care in Asian patients with previously treated metastatic colorectal cancer (CONCUR): a randomized, double-blind, placebo-controlled, phase 3 trial. Lancet Oncol 16: 619-629, 2015.

LU L, QIU C, LI D, BAI G, LIANG J, YANG Q: MicroRNA-505 suppresses proliferation and invasion in hepatoma cells by directly targeting high-mobility group box 1. Life Sci 157: 12-18, 2016.

LUMAN W, SHAFFER JL: Prevalence, outcome and associated factors of deranged liver function tests in patients on home parenteral nutrition. Clin Nutr 21: 337-343, 2002.

MARIN JJ, BUJANDA L, BANALES JM: MicroRNAs and cholestatic liver diseases. Curr Opin Gastroenterol 30: 303-309, 2014.

MUNOZ-GARRIDO P, GARCIA-FERNANDEZ D BARRENA M, HIJONA E, CARRACEDO M, MARIN JJ, BUJANDA L: MicroRNAs in biliary diseases. World J Gastroenterol 18: 6189-6196, 2012.

NINOMIYA M, KONDO Y, FUNAYAMA R, NAGASHIMA T, KOGURE T, KAKZU E: Distinct microRNAs expression profile in primary biliary cirrhosis and evaluation of miR 505-3p and miR197-3p as novel biomarkers. PLoS One 8: e66086, 2013.

RACHAGANI S, MACHA MA, MENNING MS, DEY P, PAI P, SMITH LM: Changes in microRNA (miRNA) expression during pancreatic cancer development and progression in a genetically engineered KrasG12D;Pdx1Cre mouse (KC) model. Oncotarget 6: 40295-40309, 2015.

RODERBURG C, BENZ F, VARGAS CARDENAS D, KOCH A, JANSSEN J, VUCUR M: Elevated miR-122 serum levels are an independent marker of liver injury in inflammatory diseases. Liver Int 35: 1172-1184, 2015.

SHIGEHARA K, YOKOMURO S, ISHIBASHI O, MIZUGUCHI Y, ARIMA Y, KAWAHIGASHI Y: Real-time PCRbased analysis of the human bile microRNAome identifies miR-9 as a potential diagnostic biomarker for biliary tract cancer. PLoS One 6: e23584, 2011.

SCHULTZ NA, DEHLENDORFF C, JENSEN BV, BJERREGAARD JK, NIELSEN KR, BOJESEN SE: MicroRNA biomarkers in whole blood for detection of pancreatic cancer. JAMA 311: 392-404, 2014. 
VISHNUBALAJI R, HAMAM R, ABDULLA MH, MOHAMMED MA, KASSEM M, AL-OBEED O: Genome-wide mRNA and miRNA expression profiling reveal multiple regulatory networks in colorectal cancer. Cell Death Dis 6: e1614, 2015.

YAMADA H, SUZUKI K, ICHINO N, ANDO Y, SAWADA A, OSAKABE K: Associations between circulating microRNAs (miR-21, miR-34a, miR-122 and miR-451) and non-alcoholic fatty liver. Clin Chim Acta 424: 99-103, 2013.

YIN J, HOU P, WU Z, WANG T, NIE Y: Circulating miR-375 and miR-199a-3p as potential biomarkers for the diagnosis of hepatocellular carcinoma. Tumour Biol 36: 4501-4507, 2015.

ZOU ZC, DAI M, HUANG ZY, LU Y, XIE HP, LI YF: MicroRNA-139-3p suppresses tumor growth and metastasis in hepatocellular carcinoma by repressing ANXA2R. Oncol Res 2018. doi: 10.3727/096504018X15178798885361.

ZHAO Y, WANG Y, WANG Y: Up-regulated miR-500a enhances hepatocarcinoma metastasis by repressing PTEN expression. Biosci Rep 37: pii: BSR20170837, 2017. 